\title{
Psychosocial aspects of rosacea with a focus on anxiety and depression
}

This article was published in the following Dove Press journal:

Clinical, Cosmetic and Investigational Dermatology

\author{
Monika Heisig' \\ Adam Reich ${ }^{2}$ \\ 'Department of Cosmetology, \\ The College of Physiotherapy in \\ Wroclaw, Wroclaw, ${ }^{2}$ Department of \\ Dermatology, University of Rzeszow, \\ Rzeszów, Poland
}

Background: Rosacea is a common, chronic skin condition characterized by facial redness and inflammatory lesions. The disease can lead to social stigmatization and may significantly reduce the quality of life of patients. Psychosocial impact of rosacea can be severe and debilitating; however, it is still underestimated.

Objective: This paper provides a literature review focused on depression and anxiety in patients with rosacea.

Conclusion: Rosacea patients have an increased risk of developing depression and anxiety and tend to avoid social situations. However, there are still limited data on this condition. Effective treatment of clinical symptoms brings significant improvement in psychological symptoms. Further studies should be conducted to investigate in more detail the psychological impact of rosacea. In addition, improvement of the efficacy of rosacea treatment is still needed.

Keywords: rosacea, depression, anxiety, quality of life, psychosocial distress

\section{Introduction}

Rosacea is a chronic skin condition characterized by erythema, inflammatory papules, pustules and telangiectasias, which typically occur on the face of middle-aged adults, especially fair skinned. ${ }^{1,2}$ The disease affects up to $22 \%$ of the general population. ${ }^{3}$ The etiology of rosacea is multifactorial, but still not fully understood; however, genetic background seems to play a major role in the pathogenesis of this skin condition. ${ }^{1}$ There are four subtypes of rosacea created by the National Rosacea Society, namely erythematotelangiectatic, papulopustular, phymatous and ocular form. ${ }^{4}$

In the past, psychiatric illnesses and alcohol abuse were commonly associated with rosacea, and this fact explains why the disease still leads to social stigma. ${ }^{5}$ Psychosocial effect of the disease can be severe and debilitating. It has already been described that rosacea can be associated with embarrassment, emotional distress, low self-esteem and avoidance of social situations. All these factors can lead to psychiatric disorders such as depression and anxiety. ${ }^{6}$ However, data on the impact of rosacea on the mental condition of patients are still very limited. Here, we have reviewed current literature and summarized our knowledge about the impact of rosacea on depression and anxiety.

\section{Methods}

A search of the CINAHL (Cumulative Index to Nursing and Allied Health Literature) as well as MEDLINE database via PubMed search engine was conducted using the following search terms: "rosacea" and "depression" or "anxiety". The identified publications were reviewed and analyzed in terms of the relevance to the topic. Our search was
Department of Dermatology, University of Rzeszów, UI. Szopena 2, 35-055

Rzeszów, Poland

Tel +4860507 6722

Email areich@ur.edu.pl 
limited to peer-reviewed journals. A total of 93 records were identified (CINAHL=68 and MEDLINE=25). After removal of duplicates, articles not related to the study aim and review papers, 13 papers were included in final analysis.

\section{Psychosocial distress in rosacea: depression and anxiety}

Facial appearance plays an enormous role in our self-esteem and interactions with other people. Thus, it is not difficult to understand why rosacea patients frequently experience fear of social judgment. Surprisingly, there are still very limited studies on the impact of rosacea on psychosocial condition of patients when compared with other common, chronic skin diseases such as acne, psoriasis, vitiligo or atopic dermatitis. $^{7-12}$ The negative impact of rosacea on psychosocial well-being and overall quality of life seems to be underestimated by clinicians. ${ }^{13}$ However, psychological factors, such as stress and anxiety, may even aggravate flushing in rosacea, leading to a vicious circle. It further supports the importance of considering not only clinical presentation but also the psychological status of patients with rosacea. ${ }^{14}$

A wrong judgment still exists in a society that the disease is a result of alcohol abuse or violent temperament, thus being a mark of laboring class or simply a betrayal of the person who blushes. ${ }^{5}$ To the best of our knowledge, there is only one study in English literature evaluating different aspects of rosacea which contribute to the feeling of stigmatization. ${ }^{6}$ In this survey including 807 participants, 30\% reported significant level of stigmatization due to rosacea. ${ }^{6}$ Importantly, men reported feelings of stigmatization more frequently than women, probably because they suffer from more severe forms of rosacea. ${ }^{6}$ The frequency of perceived stigmatization was highest among patients between 18 and 24 years old and in those who were urban residents. Interestingly, risk of stigmatization was found to be higher among patients with positive family history of rosacea. In addition, patients with reported feelings of stigmatization were more likely to avoid social interactions and had a higher rate of depression $(36.7 \%$ vs $21.1 \%, p<0.001){ }^{6}$

Comorbidity between major depressive disease and rosacea was confirmed in another study conducted by Gupta et $\mathrm{al}^{15}$ from 1995 to $2002 .{ }^{16} \mathrm{Few}$ other studies also analyzed the impact of rosacea on the development of depressive symptoms. ${ }^{17-20}$ However, according to the study by Abram et al, ${ }^{18}$ the presence of depressive symptoms is probably not related to the severity of the disease but rather to the subjective rosacea perception by the patients. In contrast, depression itself does not constitute a risk factor for the development of rosacea. ${ }^{19}$ Recently, in a Danish nationwide study, it was shown that mild and moderate-to-severe rosacea increased the risk of both depression (incidence rate ratios [IRR] 1.89 [95\% CI 1.82-1.96] and IRR 2.04 [95\% CI 1.96-2.12], respectively) and anxiety disorders (IRR 1.80 [95\% CI 1.75-1.86] and IRR 1.98 [95\% CI 1.91-2.05], respectively). ${ }^{20}$

In the study of Bewley et al, ${ }^{21}$ it was shown that facial erythema in rosacea caused significantly more impairment of health-related quality of life (HRQoL) than inflammatory lesions. Furthermore, rosacea patients often demonstrate low self-esteem ${ }^{22}$ and present a higher incidence of anxiety compared with the rest of the population. ${ }^{23}$ Recurrent flushing seems to play an important role in developing anxiety among rosacea patients. ${ }^{2}$ Fear of blushing may lead to social anxiety. Transient erythema could be misinterpreted by other people as an intense emotional response, and, as a coping mechanism, patients with rosacea may avoid social situations. ${ }^{17}$ In some patients, anxiety may even resemble panic disorders. ${ }^{22}$ Rosacea individuals may also feel that their facial defect diminishes their sexual attractiveness and limits career development. ${ }^{6,22}$ In a recent study by Wu et al, ${ }^{23}$ significantly higher Dermatology Life Quality Index (DLQI), anxiety and depression scores were observed in the rosacea group compared to the control group. Total DLQI score of patients was positively related to anxiety and depression scores. ${ }^{23}$

As already mentioned, erythema seems to have a major implication on patient's HRQoL. However, it is difficult to provide in-depth characteristics of HRQoL impairment among rosacea patients, as there are just few studies analyzing this aspect of rosacea. Second, different questionnaires are being chosen between various studies. The rosacea-specific quality of life questionnaire is a validated 21 -item form covering three aspects of the disease: symptomatic, emotional and functional. ${ }^{24}$ Using this questionnaire in a cross-sectional analysis of 135 patients, it was shown that phymatous form of rosacea is causing overall more HRQoL impairment than other rosacea subtypes. ${ }^{25}$ Furthermore, rosacea is causing as much reduction of HRQoL level as other chronic skin conditions, such as vitiligo, occupational dermatitis and leg ulcers. ${ }^{25}$ In another study evaluating the association between DLQI scoring and anxiety and depression symptoms in rosacea, symptoms of anxiety and depression were indirectly linked with somatic symptoms, mediated through quality of life level and stigmatization feeling. ${ }^{26}$ Remarkably, men were more negatively affected by the disease than women. ${ }^{26}$ This result again indicates a more severe psychological effect of rosacea among male gender, despite that female tend to suffer from the disease more often. In Table 1, we have summarized currently available data on depression and anxiety in patients with rosacea. 
Table I Summary of studies focusing on impact of rosacea on stigmatization, quality of life, anxiety and depression

\begin{tabular}{lll}
\hline Study & Sample size & Methods \\
\hline Gupta et al, 2005'5 & Over I3.9 million patients & - ICD-9-CM codes \\
& & \\
& & \\
Abram et al, 200918 & 70 patients attending a & - VAS assessing how \\
& $\begin{array}{l}\text { dermatologist (seekers) } \\
\text { and } 56 \text { with rosacea }\end{array}$ & disturbing rosacea was \\
& selected randomly from the & - Estonian Mood Scale \\
population (non-seekers) & questionnaire \\
& & \\
Bewley et al, & 5 randomized controlled & - DLQI \\
trials & I open-label safety study & - FRQ \\
& I epidemiological study & - PSLQ
\end{tabular}

Spoendlin et al, $2014^{19}$

Moustafa et al, $\quad 17$ studies $2014^{24}$

Su and Drummond, 31 patients $2012^{17}$

Chodkiewicz et al, $2007^{16}$

Bohm et al, 201426 168 patients
Case-control study among 53,927 rosacea patients

40 people with rosacea and 40 controls
UK-based General Practice Research Database

A search of the MEDLINE, EMBASE and psychINFO databases

- Blushing Propensity Scale,

- the Fear of Negative

Evaluation Questionnaire

- the Phobia Scale

- Depression Anxiety and

Stress Scale

- the Social Interaction

Anxiety Scale

- the Social Phobia Scale

Details not available

- DLQI,

- the rejection scale of the Questionnaire on

Experience with Skin

Complaints (QES)

- HADS
Major findings

$94,521(0.68 \%)$ rosacea patients also had a diagnosis of a depressive disease, which constitutes $\sim 70 \%$ of all psychiatric comorbidities among rosacea patients. This was much higher than the $29.9 \%$ prevalence of depression among all psychiatric patient visits.

Seekers presented significantly higher VAS scores $(6.2 \pm 3.1)$

compared with non-seekers $(3.1 \pm 2.7)(p<0.00 I)$.

Higher mean VAS scores were associated with the presence of depressive symptoms, but not with the disease severity.

Health care-seeking behavior was associated with higher subjective disease perception.

Regarding EQ5D index, domains of pain/discomfort (31.5\% moderate or extreme pain) and anxiety/depression (26.4\% moderate or extreme) were mostly affected.

Almost half (43\%) of the subjects had at least moderately impaired HRQoL, including $19.8 \%$ with a DLQI total score of $\geq 10$ points indicating severely impaired HRQoL.

Among subjects self-assessing their rosacea as severe, almost two-thirds (62\%) reported that their erythema of rosacea at least somewhat interfered with their social life and almost half (47.8\%) with their work life.

Erythema of rosacea causes a marked decrease of HRQoL in most patients, especially those with self-perceived severe erythema and without inflammatory lesions.

Depression or other affective disorders were not associated with incident rosacea, whereas patients with schizophrenia were at a decreased risk of rosacea.

A decreased risk of rosacea among people with chronic lithium exposure was observed.

Patients with rosacea have higher incidences of embarrassment, social anxiety, depression and decreased quality of life compared with the rest of the population.

Blushing propensity scores were elevated in people with severe rosacea.

Fear of blushing may contribute to social anxiety and avoidance. Cognitive-behavioral therapy for fear of blushing may help to reduce social anxiety in people with severe rosacea.

Participants with extensive facial papules and pustules had higher blushing propensity, stress and social phobia scores than controls or those without papules or pustules.

Cognitive-behavioral therapy appeared to be helpful for managing social anxiety in some individuals with rosacea with a fear of blushing.

Patients with rosacea were less satisfied with their lives, subjectively felt that they receive poor social support, had difficulty in functioning in everyday life, worse general health perception, along with a higher level of anxiety and depression. Level of anxiety and depression and social integration were proved to be the predictors of life satisfaction. Symptoms of anxiety and depression were indirectly linked with somatic symptoms, mediated through quality of life and stigmatization. 
Table I (Continued)

\begin{tabular}{llll}
\hline Study & Sample size & Methods & Major findings \\
\hline $\begin{array}{l}\text { Egeberg et al, } \\
2016^{20}\end{array}$ & $4,632,34 I$ people & Nationwide registers & $\begin{array}{l}\text { Mild and moderate-to-severe rosacea increased the risk of both } \\
\text { depression and anxiety disorders. } \\
\text { Wu et al, 20I723 }\end{array}$ \\
& $\begin{array}{l}\text { 20I rosacea patients, 196 } \\
\text { controls }\end{array}$ & - HADS & Patients with rosacea had significantly higher level of depression \\
& & & $\begin{array}{l}\text { and anxiety. } \\
\text { Quality of life impairment was significantly related to anxiety and } \\
\text { depression scores. }\end{array}$
\end{tabular}

Abbreviations: DLQI, Dermatology Life Quality Index; EQ5D, EuroQol-5D; FRQ, Facial Redness Questionnaire; HADS, Hospital Anxiety and Depression Scale; HRQoL, health-related quality of life; PSLQ, Productivity and Social Life Questionnaire; VAS, visual analog scale.

\section{Treatment of psychological symptoms}

Different studies confirm that appropriate treatment apart from clinical improvement also brings improvement in psychological symptoms and HRQoL of rosacea patients. ${ }^{24}$ In the past few decades, there have been several advances in rosacea treatment. However, options for treating erythema, especially in the case of flushing, are still limited. New treatment methods, such as laser therapy (eg, pulsed dye laser and diode laser) or topical $0.33 \%$ brimonidine gel, are still not fully satisfactory for many patients. ${ }^{27-30}$ Intradermal botulinum toxin injection on refractory erythema and rosacea flushing can also be beneficial; however, further investigations are needed. ${ }^{31}$ In some cases, psychological help, such as cognitive-behavioral therapy, may be considered as a helpful method for managing social anxiety. ${ }^{17}$ However, in fact, any treatment which targets fear of blushing may help to reduce social anxiety and depressive mood in people with rosacea. ${ }^{17}$ Different studies confirmed that currently available methods of rosacea treatment improve to some extent HRQoL, ${ }^{32-34}$ and, by extrapolation, they should also bring improvement in psychological well-being, although more detailed data on this matter are still needed.

\section{Conclusion}

Psychosocial effect of rosacea can be severe and debilitating and lead to social anxiety and depression. Men tend to experience more severe psychological impairment compared to women. The problem of stigmatization and psychosocial distress in rosacea is still underestimated, and it is important for clinicians to acknowledge the psychological impact of this disease to stimulate them to introduce more comprehensive treatment. Psychological support may be considered as an additional therapy to medical treatment as it may improve patient's outcomes. All treatment methods that lead to improvement of rosacea symptoms result in better quality of life of patients.

\section{Disclosure}

The authors report no conflicts of interest in this work.

\section{References}

1. Picardo M, Eichenfield LF, Tan J. Acne and rosacea. Dermatol Ther. 2017;7:43-52.

2. Baldwin HE. Psychosocial implications of rosacea. Dermatologist. 2012;(Suppl):2-4.

3. Tan J, Berg M. Rosacea: current state of epidemiology. J Am Acad Dermatol. 2013;69(6 suppl 1):S27-S35.

4. Wilkin J, Dahl M, Detmar M, et al. Standard classification of rosacea: report of the National Rosacea Society expert committee on the classification and staging of rosacea. J Am Acad Dermatol. 2002;46(4):584-587.

5. Cribier B. The red face: art, history and medical representations. Ann Dermatol Venereol. 2011;138(Suppl 3):S172-S178.

6. Halioua B, Cribier B, Frey M, Tan J. Feeling of stigmatization in patients with rosacea. J Eur Acad Dermatol Venereol. 2017;31(1):163-168.

7. Ginsburg IH, Link BG. Feelings of stigmatization in patients with psoriasis. J Am Acad Dermatol. 1989;20(1):53-63.

8. Hrehorow E, Salomon J, Matusiak L, Reich A, Szepietowski JC. Patients with psoriasis feel stigmatized. Acta Derm Venereol. 2012;92(1):67-72.

9. Schmid-Ott G, Kuensebeck HW, Jaeger B, et al. Validity study for the stigmatization experience in atopic dermatitis and psoriatic patients. Acta Derm Venereol. 1999;79(6):443-447.

10. Fried RG. Acne vulgaris: the psychosocial and psychological burden of illness. Dermatologist. 2013;21:2773.

11. Ongenae K, Dierckxsens L, Brochez L, van Geel N, Naeyaert JM. Quality of life and stigmatization profile in a cohort of vitiligo patients and effect of the use of camouflage. Dermatology. 2005;210(4):279-285.

12. Schmid-Ott G, Kunsebeck HW, Jecht E, et al. Stigmatization experience, coping and sense of coherence in vitiligo patients. J Eur Acad Dermatol Venereol. 2007;21(4):456-461.

13. Cardwell L, Farhangian M, Alinia H, Kuo S, Feldman S. Psychological disorders associated with rosacea: analysis of unscripted comments. J Dermatol Derm Surg. 2015;19:99-103.

14. Dirschka T, Micali G, Papadopoulos L, Tan J, Layton A, Moore S. Perceptions on the psychological impact of facial erythema associated with rosacea: results of international survey. Dermatol Ther (Heidelb). 2015;5(2):117-127.

15. Gupta MA, Gupta AK, Chen SJ, Johnson AM. Comorbidity of rosacea and depression: an analysis of the National Ambulatory Medical Care Survey and National Hospital Ambulatory Care Survey-Outpatient Department data collected by the U.S. National Center for Health Statistics from 1995 to 2002. Br J Dermatol. 2005;153(6):1176-1181.

16. Chodkiewicz J, Salamon M, Miniszewska J, WozniackaA. Psychospołeczne aspekty funkcjonowania osób chorych na trądzik różowaty [Psychosocial impact of rosacea]. Przegl Lek. 2007;64(12):997-1001. Polish.

17. Su D, Drummond PD. Blushing propensity and psychological distress in people with rosacea. Clin Psychol Psychother. 2012;19(6):488-495. 
18. Abram K, Silm H, Maaroos HI, Oon M. Subjective disease perception and symptoms of depression in relation to healthcare-seeking behaviour in patients with rosacea. Acta Derm Venereol. 2009;89(5): 488-491.

19. Spoendlin J, Bichsel F, Voegel J, Jick SS, Meier CR. The association between psychiatric diseases, psychotropic drugs and the risk of incident rosacea. Br J Dermatol. 2014;170(4):878-883.

20. Egeberg A, Hansen PR, Gislason GH, Thyssen JP. Patients with rosacea have increased risk of depression and anxiety disorders: a Danish Nationwide Cohort Study. Dermatology. 2016;232(2):208-213.

21. Bewley A, Fowler J, Schofer H, Kerrouche N, Rives V. Erythema of rosacea impairs health-related quality of life: results of a meta-analysis. Dermatol Ther (Heidelb). 2016;6(2):237-247.

22. Blount BW, Pelletier AL. Rosacea: a common, yet commonly overlooked condition. Am Fam Physician. 2002;66(3):435-440.

23. Wu Y, Fu C, Zhang W, Li C, Zhang J. The dermatology life quality index (DLQI) and the hospital anxiety and depression (HADS) in Chinese rosacea patients. Psychol Health Med. Epub 2017 Aug 10.

24. Moustafa F, Lewallen RS, Feldman SR. The psychological impact of rosacea and the influence of current management options. J Am Acad Dermatol. 2014;71(5):973-980.

25. Kini SP, Nicholson K, DeLong LK, et al. A pilot study in discrepancies in quality of life among three cutaneous types of rosacea. J Am Acad Dermatol. 2010;62(6):1069-1071.
26. Bohm D, Schwanitz P, Stock Gissendanner S, Schmid-Ott G, Schulz W. Symptom severity and psychological sequelae in rosacea: results of a survey. Psychol Health Med. 2014;19(5):586-591.

27. Odom R, Dahl M, Dover J, et al; National Rosacea Society Expert Committee on the Classification and Staging of Rosacea. Standard management options for rosacea, part 2: options according to subtype. Cutis. 2009;84(2):97-104.

28. Shanler SD, Ondo AL. Successful treatment of the erythema and flushing of rosacea using a topically applied selective alpha1-adrenergic receptor agonist, oxymetazoline. Arch Dermatol. 2007;143(11):1369-1371.

29. Clark SM, Lanigan SW, Marks R. Laser treatment of erythema and telangiectasia associated with rosacea. Lasers Med Sci. 2002;17(1):26-33.

30. Drummond PD, Su D. Blushing in rosacea sufferers. J Psychosom Res. 2012;72(2):153-158.

31. Park K, Hyun M, Jeong S, et al. Botulinum toxin for the treatment of refractory erythema and flushing rosacea. Dermatology. 2015;230(4): 299-301.

32. Shim TN, Abdullah A. The effect of pulsed dye laser on the Dermatology Life Quality Index in erythematotelangiectatic rosacea patients: an assessment. J Clin Aesthet Dermatol. 2013;6(4):30-32.

33. Menezes N, Moreira A, Mota G, Baptista A. Quality of life and rosacea: pulsed dye laser impact. J Cosmet Laser Ther. 2009;11(3):139-141.

34. Tan SR, Tope WD. Pulsed dye laser treatment of rosacea improves erythema, symptomatology, and quality of life. J Am Acad Dermatol. 2004;51(4): 592-599.
Clinical, Cosmetic and Investigational Dermatology

\section{Publish your work in this journal}

Clinical, Cosmetic and Investigational Dermatology is an international, peer-reviewed, open access, online journal that focuses on the latest clinical and experimental research in all aspects of skin disease and cosmetic interventions. This journal is included on PubMed. The manuscript management system is completely online

\section{Dovepress}

and includes a very quick and fair peer-review system, which is all easy to use. Visit http://www.dovepress.com/testimonials.php to read real quotes from published authors 